\title{
Atrial natriuretic peptide gene transfection with a novel envelope vector system ameliorates pulmonary hypertension in rats
}

Yoshihide Mitani, MD, PhD, ${ }^{\text {a }}$ Junko Maruyama, MD, PhD, ${ }^{\mathrm{b}}$ Bao Hua Jiang, MD, PhD, ${ }^{c}$ Hirofumi Sawada, MD, PhD, ${ }^{a}$ Hideto Shimpo, MD, PhD, ${ }^{d}$ Kyoko Imanaka-Yoshida, MD, PhD, ${ }^{\text {e }}$ Yasufumi Kaneda, MD, PhD, ${ }^{f}$ Yoshihiro Komada, MD, PhD, and Kazuo Maruyama, MD, $\mathrm{PhD}^{\mathrm{c}}$

From the Departments of Pediatrics, ${ }^{\text {a }}$ Physiology, ${ }^{\mathrm{b}}$ Anesthesiology, ${ }^{\mathrm{c}}$ Thoracic Cardiovascular Surgery, ${ }^{\mathrm{d}}$ Pathology, ${ }^{\mathrm{e}}$ Mie University Graduate School of Medicine, Tsu, Mie, Japan, and the Department of Molecular Therapy, ${ }_{\mathrm{f}}^{\mathrm{f}}$ Osaka University Graduate School of Medicine, Suita, Osaka, Japan.

Supported in part by grants 12470319 , 13877256, 14370484, 16390449 (to KM) from the Ministry of Education, Science, and Culture of Japan. Dr Jiang is supported by FY2002 JSPS (Japan Society for the Promotion of Science) postdoctoral fellowship for foreign researcher.

Received for publication July 30, 2007; revisions received Oct 8, 2007; accepted for publication Oct 19, 2007.

Address for reprints: Yoshihide Mitani, MD, $\mathrm{PhD}$, Department of Pediatrics, Mie University Graduate School of Medicine, 2-174 Edobashi, Tsu City, Mie Prefecture, 5148507, Japan (E-mail: ymitani@clin.medic. mie-u.ac.jp).

J Thorac Cardiovasc Surg 2008;136:142-9 $0022-5223 / \$ 34.00$

Copyright $(2008$ by The American Association for Thoracic Surgery

doi:10.1016/j.jtcvs.2007.10.061
Objectives: A novel hemagglutinating virus of Japan (HVJ, a murine parainfluenza virus) envelope vector system, in which DNA is incorporated into an inactivated viral particle deprived of its genome, was recently developed as a ready-to-use vector for gene therapy. We therefore investigated whether intratracheal gene transfer using this vector can induce transgene expression in the lung and whether atrial natriuretic peptide gene transfer ameliorates pulmonary hypertension in rats.

Methods: Rats transfected intratracheally with $\beta$-galactosidase vector, atrial natriuretic peptide vector, or mock vector were investigated for the evaluation of $\beta$-galactosidase expression, atrial natriuretic peptide mRNA expression, and inflammatory cell infiltration. Rats were divided into 5 treatment groups $(\mathrm{n}=73)$ : normoxic rats treated intratracheally with mock vector or atrial natriuretic peptide gene and chronic hypoxic rats treated similarly with mock vector, atrial natriuretic peptide, or a reporter gene, $\beta$-galactosidase. Pulmonary hypertension and transfected gene expression were evaluated.

Results: $\beta$-Galactosidase gene transfer induced its intense enzymatic activity in bronchial and alveolar epithelial cells but not in other organs in normoxic rats. Transfected lungs were not associated with inflammatory cell infiltration. Atrial natriuretic peptide gene transfection inhibited pulmonary hypertension, which is associated with its mRNA expression in the lungs. Indices of right ventricular hypertrophy and pulmonary vascular diseases induced by chronic hypoxia were significantly but incompletely ameliorated.

Conclusions: HVJ-envelope vector is an efficient, relatively safe, and ready-to-use gene delivery system for pulmonary vascular diseases. Atrial natriuretic peptide gene transfer to lungs by using this vector could be a promising therapeutic approach against pulmonary hypertension.

$\mathrm{T}$ The development of efficient and safe vector systems is a major challenge in the clinical application of gene therapy against pulmonary hypertension. Initial gene transfer experiments for the lungs, performed by using adenoviral vectors, provoked significant inflammatory responses, which limited the duration of gene expression in animal models ${ }^{1,2}$ and their clinical utility in human trials. ${ }^{3,4}$ The delivery of other current viral vectors (retroviral and adeno-associated viral vectors) results in the incorporation of the exogenous DNA into the host genome, which raises concerns for the development of malignant disorders. ${ }^{5,6}$ Recently, a novel hemagglutinating virus of Japan (HVJ, a murine parainfluenza virus) envelope vector system was developed, in which plasmid DNA was incorporated into the lipid envelope of the inactivated virus deprived of the viral genome. ${ }^{7}$ Although previous nonviral vectors have limited transfection efficiency in vivo, this gene delivery system confers high transfection efficiency in vivo and in vitro by means of a specific HVJ-cell fusion 


\section{Abbreviations and Acronyms \\ ANP $=$ atrial natriuretic peptide \\ $\mathrm{LV}+\mathrm{S}=$ left ventricle plus septum \\ PA $=$ pulmonary artery \\ PBS = phosphate-buffered saline \\ $\mathrm{RV}=$ right ventricle \\ VCAM-1 = vascular cell adhesion molecule 1}

machinery. ${ }^{7}$ In addition, this is a new ready-to-use vector for gene therapy, which is more easily prepared compared with the previous HVJ-liposome vector. ${ }^{8}$ However, the biologic relevance of this transfection system has not been established in the lungs, and intratracheal gene transfer with this system has not been applied to gene transfer experiments against pulmonary hypertension.

Accumulated evidence suggests that atrial natriuretic peptide (ANP) plays a pivotal role in counteracting the mechanisms involved in the development of pulmonary hypertension. Importantly, pulmonary vasodilator responses to ANP are well preserved or exaggerated in pulmonary hypertension. ${ }^{9,10}$ Moreover, treatments that increase ANP levels, either by means of ANP supplementation or inhibition of the neutral endopeptidase that degrades ANP in vivo, attenuate pulmonary hypertension and vascular remodeling. ${ }^{11,12}$ Therefore ANP gene transfer to the lungs is warranted as a therapeutic approach against pulmonary hypertension.

We therefore investigated whether efficient gene transfer to the lungs can be conferred by the novel HVJ-envelope vector system and whether this system is of biologic relevance in inhibiting the development of pulmonary hypertension and vascular diseases in rats by means of ANP gene transfer.

\section{Materials and Methods \\ Plasmids, Preparation of HVJ-envelope Vector, and Intratracheal Gene Transfer}

The expression vector for rat ANP was constructed as previously described. ${ }^{13}$ In brief, a fragment containing rat ANP cDNA was inserted into the $\mathrm{XhoI}$ site of the pUC-CAGGS expression vector plasmid. The expression vector for the $\beta$-galactosidase gene (Lac $Z$ ) was similarly constructed. In this plasmid transcription of the ANP or Lac Z cDNA was under the control of the chicken $\beta$-actin promoter and the cytomegalovirus enhancer. It has been verified that cells transfected with the pUC/ANP construct release biologically active ANP peptide into the cell culture medium. ${ }^{13} \mathrm{HVJ}$-envelope vector was prepared as described previously. ${ }^{7}$ Briefly, the virus suspension $(10,000$ hemagglutinating units) was inactivated by means of UV irradiation $\left(99 \mathrm{~mJ} / \mathrm{cm}^{2}\right)$ and mixed with plasmid DNA $(200 \mu \mathrm{g})$ and $0.3 \%$ Triton-X. After centrifugation, it was washed with $1 \mathrm{~mL}$ of balanced salt solution $(10 \mathrm{mmol} / \mathrm{L}$ Tris- $\mathrm{Cl}$ [pH 7.5], $137 \mathrm{mmol} / \mathrm{L} \mathrm{NaCl}$, and $5.4 \mathrm{mmol} / \mathrm{L} \mathrm{KCl}$ ) to remove the detergent and unincorporated DNA. After centrifugation, the envelope vector was suspended in $100 \mu \mathrm{L}$ of phosphate-buffered saline (PBS). The vector was stored at $4^{\circ} \mathrm{C}$ until use. HVJ-envelope vector solution $(0.2 \mathrm{~mL})$ including $50 \mu \mathrm{g}$ of Lac Z or ANP gene or the empty vector solution was injected through a 24-gauge intravascular catheter (TERUMO, Tokyo, Japan) into the trachea, which is pre-exposed by means of a midline skin incision after achievement of pentobarbital sodium anesthesia $(33 \mathrm{mg} / \mathrm{kg}$ by means of intraperitoneal injection) while not ventilated.

\section{$\beta$-Galactosidase Expression, Rat ANP Reverse Transcriptase-Polymerase Chain Reaction, and Immunohistochemistry}

Rats 4 days after Lac Z, ANP vector, or mock transfection or not transfected through the trachea were killed after achievement of pentobarbital sodium anesthesia for the evaluation of $\beta$-galactosidase expression, ANP mRNA expression, and inflammatory cell infiltration.

The lungs were perfused with PBS through a pulmonary artery (PA) cannula, fixed with $1 \%$ glutaraldehyde in PBS through PA and tracheal cannulae at $4^{\circ} \mathrm{C}$ for 4 hours, and subsequently substituted with and immersed in cold PBS containing 30\% sucrose overnight before being embedded and frozen in Tissue-Tek OCT compound (Miles Inc., IN) to evaluate $\beta$-galactosidase expression. The X-gal assay was performed as follows. ${ }^{14}$ Cryostat sections (4- $\mu \mathrm{m}$ slice) were washed with PBS and incubated at $37^{\circ} \mathrm{C}$ for 4 hours in an X-gal solution (in PBS: $1 \mathrm{mg} / \mathrm{mL}$ X-gal [Sigma, St Louis, Mo; in dimethyl sulfoxide], $5 \mathrm{mmol} / \mathrm{L} \mathrm{K} 3 \mathrm{Fe}[\mathrm{CN}] 6,5 \mathrm{mmol} / \mathrm{L} \mathrm{K} 4 \mathrm{Fe}(\mathrm{CN}) 6$, and $2 \mathrm{mmol} / \mathrm{L} \mathrm{MgCl}_{2}$ ). The sections were washed in water, counterstained with hematoxylin, dehydrated with ethanol, and mounted to stop the enzymatic reaction. Some sections were subsequently incubated with biotinated smooth muscle $\alpha$-actin (Sigma) for 1 hour and visualized with $3^{\prime}, 3^{\prime}$-diaminobenzidine to distinguish between smooth muscle cells and $\beta$-galactosidase-positive cells.

For the evaluation of rat ANP expression, total RNA was extracted from rat lungs, which were carefully isolated from the trachea and bronchi, by using a modified single-step acid-guanidine thiocyanate-phenol-chloroform method ${ }^{15}$ with ISOGEN reagent (Nippon Gene, Tokyo, Japan). The ethanol-precipitated RNA fraction $(1 \mu \mathrm{g})$ was reverse transcribed with SuperScript II reverse transcriptase (GIBCO BRL, Gaithersburg, Md), according to the manufacturer's protocol, and $20 \mu \mathrm{L}$ of first-strand cDNA solution was obtained. Primer sequences for rat ANP were $5^{\prime}$-AAC CTG-CTA-GAC-CAC-CTG-3' and 5'-TTT-TCA-AGA-GGG CAG-ATC-3 ${ }^{\prime}{ }^{16}$ Samples were kept at $95^{\circ} \mathrm{C}$ for 10 minutes and subjected to thermocycling ( 28 cycles of 30 seconds at $95^{\circ} \mathrm{C}, 30$ seconds at $55^{\circ} \mathrm{C}$, and 45 seconds at $72^{\circ} \mathrm{C}$, with a final extension at $72^{\circ} \mathrm{C}$ for 7 minutes) to amplify ANP cDNA, generating a 214bp fragment. Primer sequences for rat $\beta$-actin (internal controls) were $5^{\prime}$-GGG-AAA-TCG-TGC-GTG-ACA-T-3' and $5^{\prime}$-CAG GAG-GAG-CAA-TGA-TCT-T- $3^{\prime}$. Samples were kept at $95^{\circ} \mathrm{C}$ for 15 minutes and subjected to thermocycling ( 25 cycles of 30 seconds at $95^{\circ} \mathrm{C}, 30$ seconds at $55^{\circ} \mathrm{C}$, and 1 minute at $72^{\circ} \mathrm{C}$, with a final extension at $72^{\circ} \mathrm{C}$ for 5 minutes) to amplify $\beta$-actin cDNA, generating a $479-\mathrm{bp}$ fragment. One microliter of a $20-\mu \mathrm{L}$ reaction was run on an agarose gel by using the DNA 7500 LabChip kit (Agilent Technologies, Tokyo, Japan).

The lungs were perfused with PBS through a PA cannula, fixed with $4 \%$ paraformaldehyde in PBS through PA and tracheal cannula at $4{ }^{\circ} \mathrm{C}$ for 3 hours, and subsequently substituted with and immersed in cold PBS containing 30\% sucrose overnight before being embedded and frozen in Tissue-Tek OCT compound to evaluate 
inflammatory cell infiltration. Five-millimeter sections were cut onto glass slides and stored at $-70^{\circ} \mathrm{C}$ until use. Tissue sections were air-dried, and nonspecific binding sites were blocked. Sections were incubated overnight at $4{ }^{\circ} \mathrm{C}$ with primary antibodies that recognize vascular cell adhesion molecule 1 (VCAM-1; $\times 1000$, mouse monoclonal 5F10, Covance, $\mathrm{NJ})$ and rat CD68 $(\times 500$, mouse monoclonal ED-1, Serotec, Kidlington, UK). ${ }^{17}$ After streptavidinbiotin amplification (LSAB2 kit, DAKO, Tokyo, Japan), the slides were incubated with $3^{\prime}, 3^{\prime}$-diaminobenzidine and counterstained with hematoxylin. For the positive controls of immunohistochemistry, rats were intratracheally injected with lipopolysaccharide (Escherichia coli lipopolysaccharide, serotype 055:B5, Sigma) solution $(6 \mathrm{mg} / \mathrm{kg}$ in $100 \mu \mathrm{L}$ of PBS) and similarly killed 24 hours after treatment.

\section{Animal Models}

Protocols for all animal experiments were approved by the Animal Care Committee of Mie University School of Medicine. Pulmonary hypertension was induced by exposure to chronic hypoxia in 7-week-old S-D rats weighing 190 to 230 g (CLEA Japan, Osaka, Japan). ${ }^{18}$ Rats were randomly assigned to one of 5 groups: those kept in ambient air and treated intratracheally with empty vector or rat ANP gene by using the HVJ-envelope vector and those exposed to hypobaric hypoxia (air at $380 \mathrm{~mm} \mathrm{Hg}$ ) for 10 days and treated similarly with empty vector, rat ANP gene, or Lac Z. Rats were transfected with $0.2 \mathrm{~mL}$ of the HVJ-envelope vector solution 3 days before hypoxic exposure. Food and water were provided ad libitum throughout the experiment. Rats were removed from the hypobaric chamber once daily for 10 minutes for weighing and normal care.

\section{Hemodynamic Measurements and Tissue Preparation}

After the period of hypoxic exposure, rats were catheterized after achievement of pentobarbital sodium anesthesia by using a closed-chest technique, as described previously in detail. ${ }^{18,19}$ Briefly, a catheter of silicone elastomer tubing $(0.31 \mathrm{~mm}$ ID and $0.65 \mathrm{~mm}$ OD) was inserted into the PA or the aorta through the right external jugular vein or the internal carotid artery, respectively. PA or aortic pressure was monitored by use of a physiologic transducer (Uniflow, Baxter), an amplifier system (AP-620G, Nihon Koden), and a monitor (Polygraph system, Nihon Koden). The catheter was passed under the skin and exteriorized at the back of the animal's neck. At 48 hours after catheterization, PA or aortic pressure was recorded in ambient air while the rat was fully conscious. The hematocrit value was determined from a $0.1-\mathrm{mL}$ blood sample. In this model values of cardiac output did not differ significantly between normoxic and hypoxic rats. ${ }^{20}$

After the hemodynamic measurements were completed, lung tissue was prepared for morphometric analysis of the vasculature, as previously described in detail. ${ }^{18,19}$ Briefly, after the rat was mechanically ventilated after achievement of pentobarbital sodium anesthesia, the lungs were perfused through a PA cannula with a hot $\left(60^{\circ} \mathrm{C}\right)$ mixture of radiopaque barium and gelatin at 1000 $\mathrm{mm} \mathrm{H}_{2} \mathrm{O}$ pressure for 5 minutes. Then the isolated lungs were distended and fixed by perfused $10 \%$ formalin through a tracheal tube at a pressure of $360 \mathrm{~mm} \mathrm{H}_{2} \mathrm{O}$ for 72 hours. A $10 \times 10 \times 5-\mathrm{mm}$ tissue block obtained from the midsection of the left lung was processed for light microscopy. Sections from paraffin blocks were stained by using the elastic Van Gieson method. The right ventricle (RV) was dissected from the left ventricle plus septum
$(\mathrm{LV}+\mathrm{S})$ and weighed separately. The weight ratios $\mathrm{RV} /(\mathrm{LV}+\mathrm{S})$ and RV/final body weight were calculated.

\section{Morphometric Analysis of PAs}

Light microscopic slides were analyzed blindly without knowledge of the treatment groups, as reported previously. ${ }^{18,19}$ Briefly, all barium-filled arteries of larger than $15 \mu \mathrm{m}$ in external diameter were assessed at $\times 400$ magnification. Each artery was first categorized according to its accompanying airway (ie, a terminal bronchus, respiratory bronchus, alveolar duct, or alveolar wall). The structure of each artery was determined as muscular (ie, with a complete medial coat of muscle), partially muscular (ie, with only a crescent of muscle), or nonmuscular (ie, with no apparent muscle). The percentage of muscular and partially muscular arteries at alveolar wall and alveolar duct levels was determined. For all muscular arteries with an external diameter of the media $\leq 200 \mu \mathrm{m}$, the distance between external and internal elastic laminae was measured at 2 points across the lumen along the shortest curvature and expressed as a percentage of medial wall thickness, which was calculated as twice the average wall thickness divided by the external diameter.

\section{Measurement of Plasma ANP Levels}

Plasma ANP levels were measured 15 days after administration of the mock vector and ANP plasmid in normoxic and hypoxic rats. Blood samples were harvested through a PA catheter after PA pressure measurements. The samples were assayed for rat ANP with an RIA kit (S-2039, Peninsula Laboratories, Calif), according to the supplier's instructions.

\section{Statistics}

Data are presented as means \pm standard error. The differences between multiple groups were compared by using a 1-way analysis of variance, followed by the Scheffe F test. An unpaired Student $t$ test was used to compare 2 groups.

\section{Results}

Expression of $\beta$-galactosidase and ANP in Rats Transfected by Using HVJ-envelope Vector

Four days after intratracheal Lac Z transfer, $\beta$-galactosidase was diffusely expressed in bronchial and alveolar epithelial cells (Figure $1, A-C$ ) but not obvious in pulmonary vascular endothelial or smooth muscle cells or in other organs tested (heart, muscle, aorta, liver, and spleen; Figure $1, B$ ). In contrast, $\beta$-galactosidase was not expressed in lungs from mock-transfected rats (data not shown).

Reverse transcriptase-polymerase chain reaction studies demonstrated that intratracheal ANP transfection induced mRNA expression in the lung on day 4. mRNA expression was not induced in mock-transfected rat lungs (Figure 1,D).

\section{VCAM-1 Expression and ED1-positive Cell Infiltration in Transfected Rat Lungs}

To determine whether an inflammatory reaction was induced in lungs by means of intratracheal HVJ-envelope vector administration, we examined ED1-positive mononuclear cell infiltration and VCAM-1 expression in the lungs of 

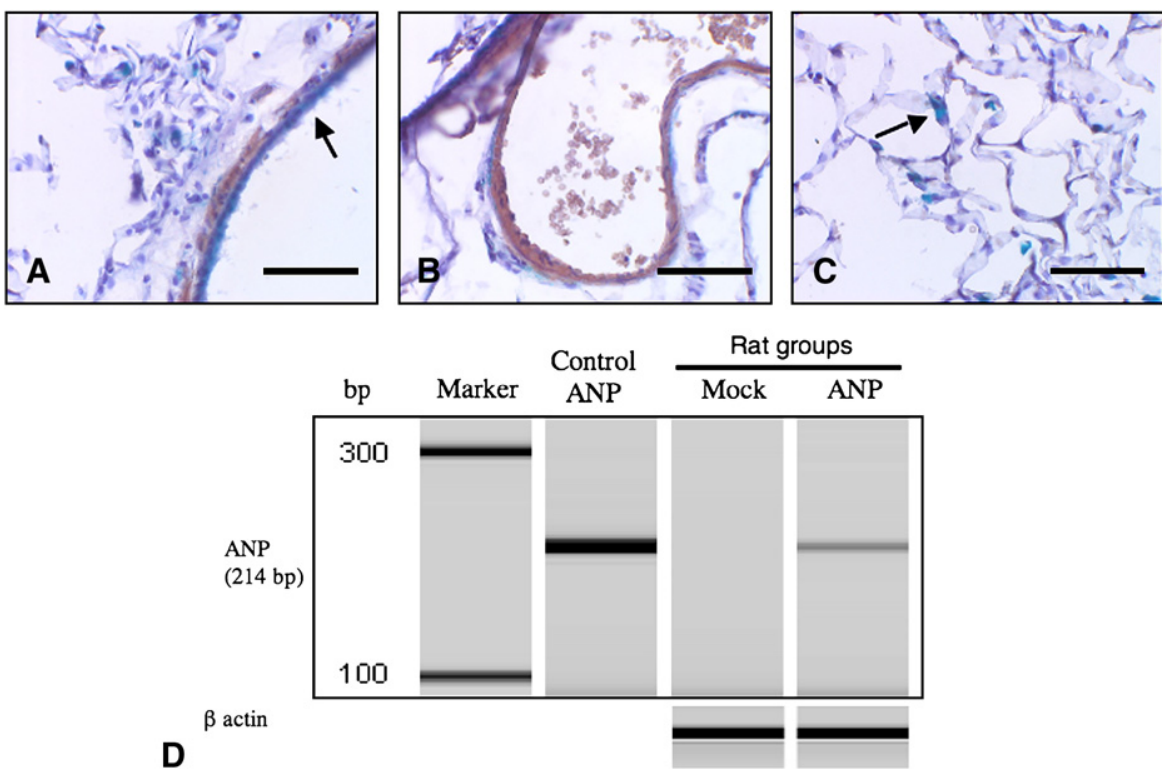

Figure 1. $\beta$-Galactosidase expression and atrial natriuretic peptide (ANP) mRNA expression were induced in rat lungs by HVJ-envelope vector-based gene transfer. $\beta$-Galactosidase expression (bright blue) was induced in rat lungs transfected through trachea ( $n=10$ ). Brown cells represent smooth muscle $\alpha$-actin-positive cells (A-C). $\beta$ Galactosidase-positive cells are found in bronchial (A) and alveolar (C) epithelial cells but not in pulmonary vascular endothelial or smooth muscle cells (B). Gene transfer with ANP vector, but not with mock vector, induced mRNA expression in the lungs, as evaluated by means of reverse transcriptase-polymerase chain reaction ( $\mathrm{n}=3 ; \mathrm{D}$ ). Marker, Size marker; control ANP, ANP gene itself used for transfection; mock (under rat groups), rats transfected with the mock vector; ANP (under rat groups), rats transfected with ANP vector. Arrowheads mark representative $\beta$-galactosidase-positive cells. Scale bars $=50 \mu \mathrm{m}$. The 214-bp band represents an ANP fragment. normoxic rats 4 days after mock transfection or nontransfection. No obvious VCAM-1 expression was observed in the pulmonary endothelium in both treatment groups (Figure 2, $A$ and $B)$. Mock transfection did not increase ED1-positive cells in the lungs (Figure 2,D,E, $G$, and $H$ ). Positive controls for the staining of VCAM-1-positive endothelium and ED-1-positive inflammatory cells were confirmed in lipopolysaccharide-treated rats (data not shown). Lack of positive staining without primary antibodies was confirmed in vector-treated lungs (Figure 2, $C, F$, and $I$ ). In addition, the findings suggestive of inflammatory lung damage (ie, foci of mild damage to alveolar or bronchial epithelial cells with edema or hemorrhage) were not found in the transfected lungs.

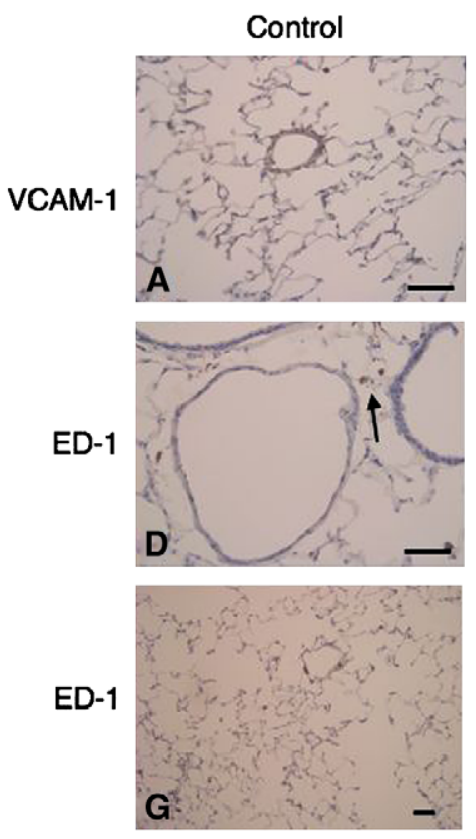

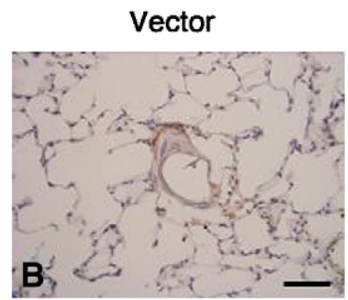
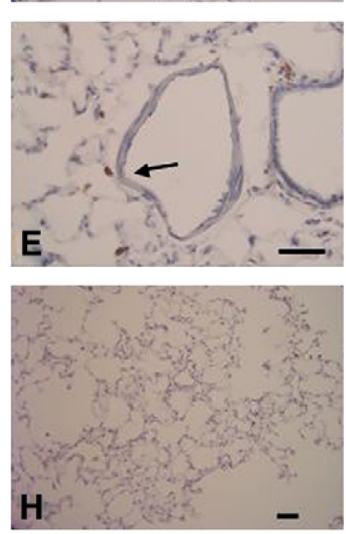

Vector $A b(-)$
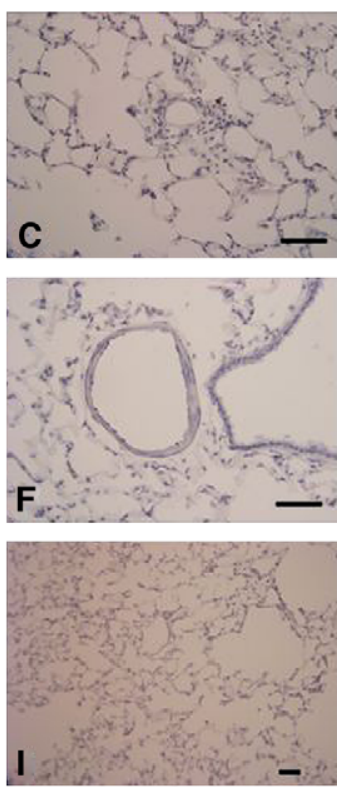

Figure 2. No obvious inflammatory responses were provoked by vector transfer with the HVJ-envelope vector. No obvious vascular cell adhesion molecule 1 (VCAM-1) expression (brown) was observed in pulmonary endothelium in either treatment group (vector or no vector; $A$ and $B$ ). Vector transfer did not increase ED1-positive cells (brown, arrows) in the lungs (D, E, G, and $H)$. Negative controls for each staining were shown (C, F, and I). Primary antibodies: VCAM-1 (A and $B$ ), ED-1 (D, E, G, and $H)$, and no antibody (C, F, and I). Bars $=50 \mu \mathrm{m} . \mathrm{N}=3$ in each treatment group. 
Effects of ANP Gene Transfer on Pulmonary Hypertension

Mean PA pressure was similar in normoxic rats treated with empty vector or ANP gene transfer. The pulmonary hypertension observed (mean PA pressure, $37.1 \pm 1.1 \mathrm{~mm} \mathrm{Hg}$ in the hypoxia/mock transfection group and $38.1 \pm 4.7 \mathrm{~mm} \mathrm{Hg}$ in the hypoxia/Lac $\mathrm{Z}$ transfection group vs $18.8 \pm 1.2 \mathrm{~mm} \mathrm{Hg}$ in the control/mock transfection group; $P>.05$, respectively) was decreased by ANP transfection $(30.8 \pm 1.1 \mathrm{~mm} \mathrm{Hg}$ vs the hypoxia/mock transfection group and the hypoxia/Lac $\mathrm{Z}$ transfection group; $P<.05$, respectively); Figure 3, $A$ ).

The $\mathrm{RV} /(\mathrm{LV}+\mathrm{S})$ ratio was similar in normoxic rats with mock or ANP gene transfection. The right ventricular hyper- tension induced by chronic hypoxia $(\mathrm{RV} /[\mathrm{LV}+\mathrm{S}]$ ratio: 38.9 \pm 0.7 in the hypoxia/mock transfection group and $37.9 \pm 1.1$ in the hypoxia/Lac $\mathrm{Z}$ transfection group vs $25.0 \pm 0.4$ in the control/mock transfection group; $P<.05$, respectively) was ameliorated by ANP transfection ( $36.5 \pm 0.8$ vs the hypoxia/ mock transfection group, $P<.05$; Figure $3, B$ ).

\section{Morphometric Analysis of PAs}

Normoxic rats with mock or ANP transfection had a similar degree of medial wall thickness. The degree of medial wall thickness increased with chronic hypoxia (mitral wall thickness: $3.82 \% \pm 0.34 \%$ in the hypoxia/mock transfection group and $4.68 \% \pm 0.42 \%$ in the hypoxia/Lac $\mathrm{Z}$ transfection

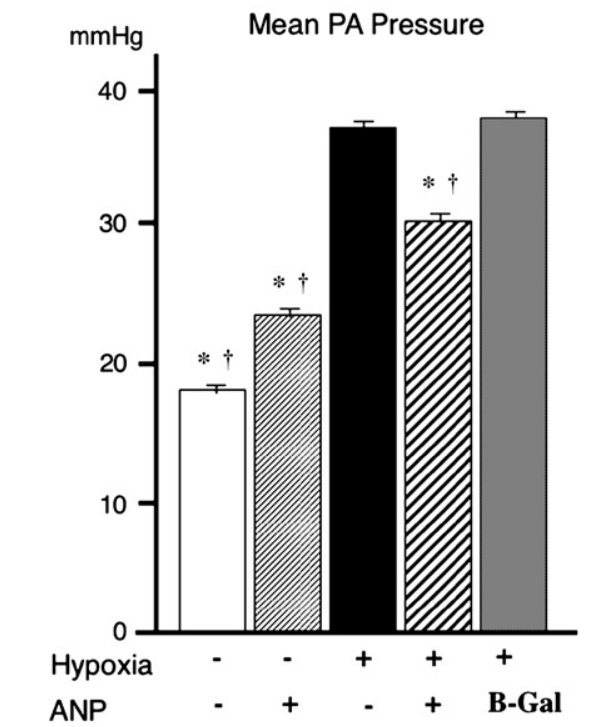

A

(13)

(8)

(13)

(15)

(9)
$\%$

\section{$\%$ Medial Wall Thickness}

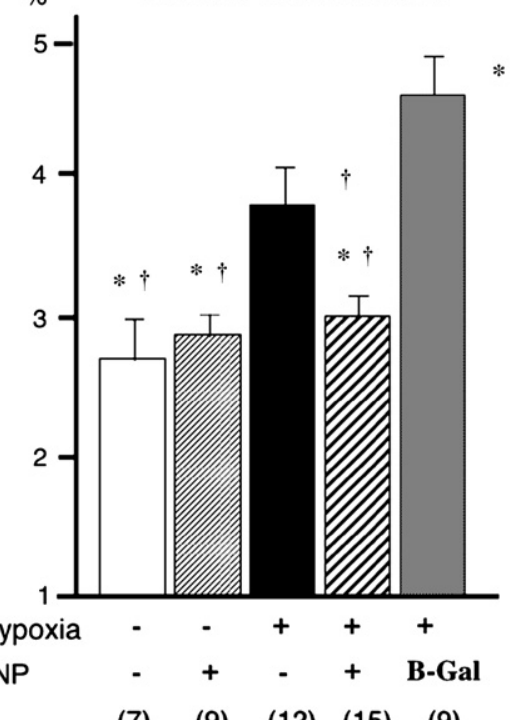

C

(7)
(9) (12) (15) (9)

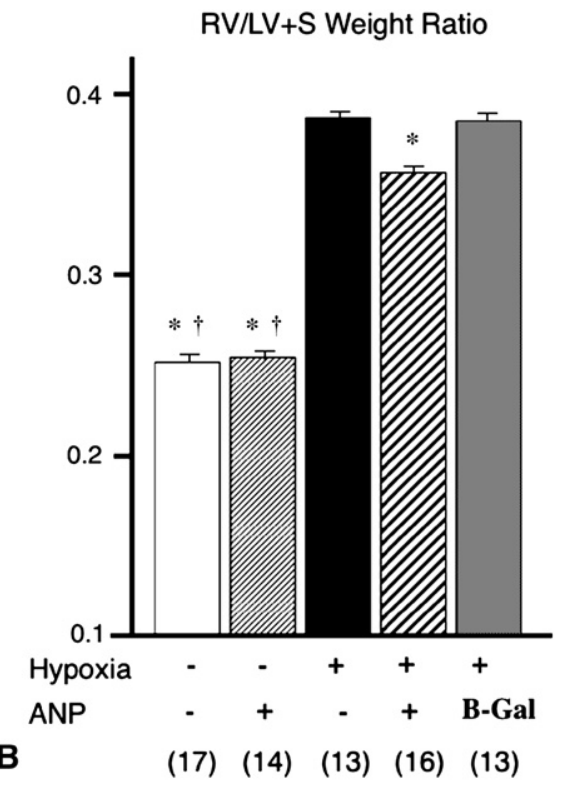

$\%$ Muscularization (Alveolar Duct level)

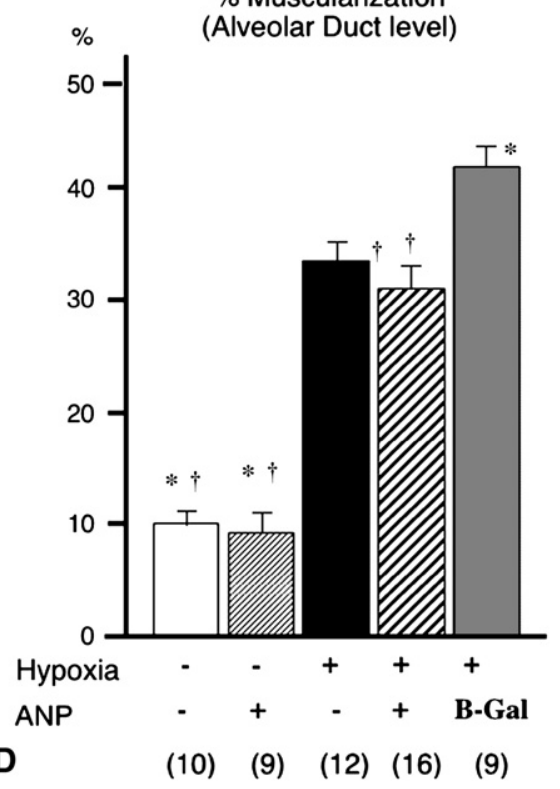

Figure 3. Atrial natriuretic peptide (ANP) gene transfer incompletely but significantly ameliorates pulmonary hypertension and vascular diseases (percentage of muscularization) in rats. ANP transfection completely inhibited the percentage of medial wall thickness. Mean pulmonary artery (PA) pressure (A), right ventricle/left ventricle plus septum $(R V / L V+S)$ weight ratio (B), percentage of medial wall thickness (C), and percentage of muscularization (D) are shown. ${ }^{*} P<.05$ versus the hypoxia/mock transfection group; $\uparrow P<.05$ versus the hypoxia/ $\beta$-galactosidase transfection group. $B$-Gal, $\beta$-Galactosidase transfection. The number of each group is described in parentheses. 
group vs $2.93 \%$ in the control/mock transfection group; $P<$ .05 , respectively) was inhibited by ANP transfection (2.95\% $\pm 0.14 \%$ vs the hypoxia/mock transfection group and the hypoxia/Lac $\mathrm{Z}$ transfection group; $P<.05$, respectively). Lac $\mathrm{Z}$ transfection increased the degree of medial wall thickness in hypoxic rats (hypoxia/Lac $\mathrm{Z}$ transfection group vs hypoxia/mock transfection group, $P<.05$; Figure $3, C$ ).

Normoxic rats with mock or ANP transfection had similar percentages of muscularization in small PA. The percentage of muscularization increased with chronic hypoxia $(32.5 \% \pm 2.7 \%$ in the hypoxia/mock transfection group and $43.6 \% \pm 2.2 \%$ in the hypoxia/Lac $\mathrm{Z}$ transfection group vs $9.7 \% \pm 1.7 \%$ in the control/mock transfection group at the alveolar duct level; $P<.05$, respectively; $27.9 \% \pm$ $3.9 \%$ in the hypoxia/mock transfection group and $34.2 \%$ $\pm 2.8 \%$ in the hypoxia/Lac $\mathrm{Z}$ transfection group vs $6.6 \%$ $\pm 1.4 \%$ in the control/mock transfection group at the alveolar wall level; $P<.05$, respectively) was ameliorated by ANP transfection $(30.7 \% \pm 2.7 \%$ vs the hypoxia/Lac $\mathrm{Z}$ transfection group at the alveolar duct level, $P<.05$; $23.2 \% \pm 2.2 \%$ vs the hypoxia/Lac $Z$ transfection group at the alveolar wall level, $P<.05$ ). Lac $\mathrm{Z}$ transfection increased the percentage of muscularization at the alveolar duct but not the alveolar wall level in hypoxic rats (hypoxia/Lac $\mathrm{Z}$ transfection group vs hypoxia/mock transfection group at the alveolar duct level, $P<.05$; Figure 3,D).

\section{Systemic Effects of ANP Gene Transfer}

Baseline plasma ANP levels in control rats were not changed by ANP transfection. Plasma ANP levels increased with chronic hypoxia $(287.5 \pm 22.7 \mathrm{pg} / \mathrm{mL}$ in the hypoxia/mock transfection group vs $136.3 \pm 22.3 \mathrm{pg} / \mathrm{mL}$ in the control/ mock transfection group, $P<.05$ ) were partially decreased by ANP transfection $(198.0 \pm 22.7 \mathrm{pg} / \mathrm{mL}$ vs the hypoxia/ mock transfection group, $P<.05$; Figure $4, A$ ).

Systolic blood pressure was not changed by hypoxic exposure or gene transfection treatment (Figure $4, B$ ). Polycythemia was induced by chronic hypoxia (hematocrit: $53.1 \% \pm 2.4 \%$ in the hypoxia/mock transfection group vs $39.3 \% \pm 1.2 \%$ in the control/mock transfection group, $P<$ .05 ; Figure $4, C$ ). Transfection of the ANP gene did not change hematocrit values in control rats (Figure 4,C). Initial body weight was similar in all 5 treatment groups. At the time of hemodynamic measurement, hypoxic rats had lower body weights than control rats $(225.6 \pm 5.3 \mathrm{~g}$ in the hypoxia/mock transfection group vs $280.6 \pm 7.3 \mathrm{~g}$ in the control/mock transfection group, $P<.05$ ). Transfection of the ANP gene or Lac $\mathrm{Z}$ did not change final body weight both in control and hypoxic rats (Figure 4,D).

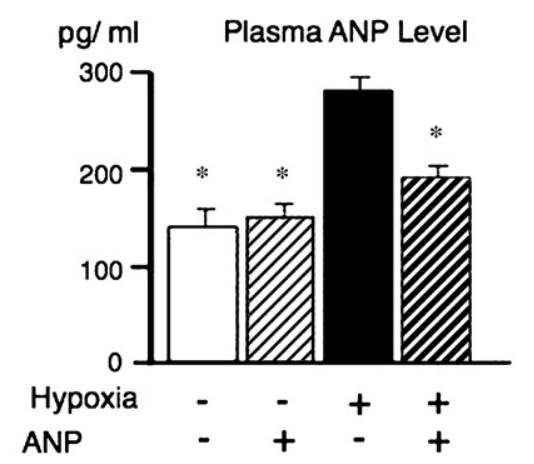

A

(4)
(5)
(4)
B

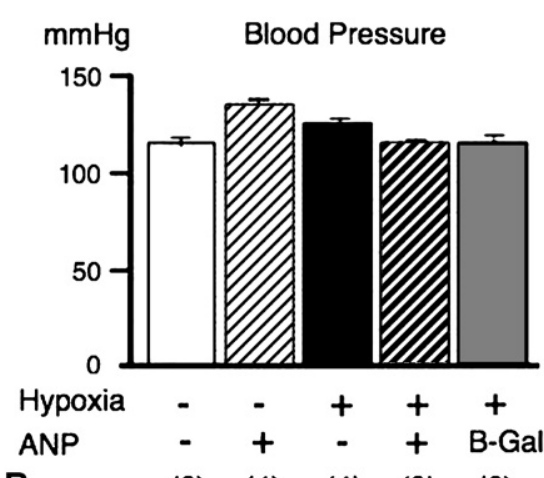

(6) (4) (4) (9) (6)

Figure 4. Systemic effects of atrial natriuretic peptide (ANP) gene transfer in rats. Plasma ANP levels (A), mean blood pressure (B), hematocrit value (C), and final body weight (D) are shown. ${ }^{*} P<.05$ versus the hypoxia/ mock transfection group; $\dagger \boldsymbol{P}<.05$ versus the hypoxia/ $\beta$-galactosidase transfection group; $\ddagger P<.05$ versus the hypoxia/ANP transfection group. $B$-Gal, $\beta$-Galactosidase transfection. The number of each group is described in parentheses.
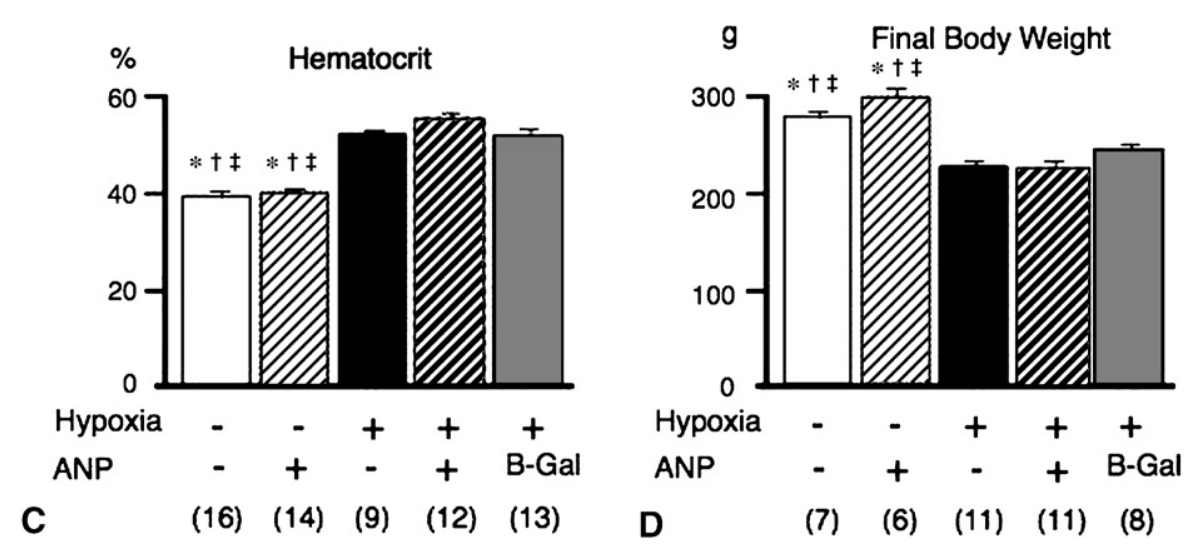

The Journal of Thoracic and Cardiovascular Surgery • Volume 136, Number 1147 


\section{Discussion}

Efficient and safe vector systems are essential to successful gene therapy against pulmonary hypertension. The present investigation demonstrates that intratracheal gene transfer with a novel HVJ-envelope vector resulted in marked transgene expression in bronchial and alveolar epithelial cells. These effects were not associated with any obvious inflammatory cell infiltration in the lungs or any transgene expression in other organs. The biologic relevance of this transfection system was further supported by the ANP gene transfer experiment, in which intratracheal gene transfer ameliorated the development of pulmonary hypertension and vascular remodeling in rats exposed to chronic hypoxia. These ameliorating effects on pulmonary vascular diseases were associated with ANP expression in the lungs, which was not accompanied by either an increase in circulating ANP levels or a decrease in blood pressure. These findings suggest that ANP gene transfer with this nonviral genome vector could be an efficient and relatively safe therapeutic strategy against pulmonary hypertension.

By using the present vector, the reporter gene transferred was intensely expressed in bronchial and alveolar epithelial cells. This HVJ-envelope vector was constructed by incorporating plasmid DNA into the inactivated HVJ viral envelope, which was deprived of the viral genome. ${ }^{7}$ The plasmid DNA is integrated into cells by means of a specific virus-cell fusion machinery without being degraded by endosomes or lysosomes. Although the present vector is one of nonviral vectors, this vector thereby overcomes the issue of the limited transfection efficiency of the previous cationic liposome for the lung by means of these specific mechanisms. ${ }^{7,21,22}$ In fact, a recent study demonstrated that compared with conventional cationic liposomes, the HVJ-envelope vector system confers 120-fold higher transfection efficiency in transfecting the uterine cavity in mice. ${ }^{7}$ The biologic relevance of this vector was demonstrated by the present experiments, in which ANP transfection ameliorated pulmonary vascular diseases in rats exposed to chronic hypoxia. These ameliorating effects were associated with ANP expression transduced in the lung. Furthermore, light microscopic and immunohistochemical studies showed that obvious inflammatory cell infiltration and endothelial VCAM-1 expression was not observed in the lungs after the transfection. These findings are consistent with the recent reports of HVJ-envelope vector-based gene transfer to other organs: the central nervous system and the uterus. ${ }^{21,22}$ One might be concerned about an increase in the percentage of mitral wall thickness and the percentage of muscularization at the alveolar duct level caused by Lac $\mathrm{Z}$ transfection in hypoxic rats. However, these effects were not consistent with those on mean PA pressure, the $\mathrm{RV} /(\mathrm{LV}+\mathrm{S})$ ratio, and the percentage of muscularization at the alveolar wall level. The levels of mean PA pressure, the $\mathrm{RV} /(\mathrm{LV}+\mathrm{S})$ ratio, the percentage of mitral wall thickness, and the percentage of muscularization in control/mock transfected rats in our findings were comparable with those in control rats in previous reports. ${ }^{18,19}$ Therefore the present unfavorable but inconsistent effects of Lac $\mathrm{Z}$ transfection, if any, might be related to Lac $\mathrm{Z}$ transfection itself and not to the HVJ-vector system. Others might be concerned about the incorporation of cDNA into the host genome in this system. Although we still do not have definitive data to negate this possibility in the HVJ-envelope vector system, it would not be the case because we previously demonstrated that the transfected gene is located in the epichromosome and not integrated in the chromosome by using the HVJ-liposome system with the similar specific HVJ-cell fusion machinery. ${ }^{23}$ Others might also be concerned about the transgene expression in prolonged time points in this model. Because transgene expression persists for at least 35 days in the previous HVJ-liposome system, ${ }^{23}$ which confers transfection similarly through the specific HVJ-cell fusion machinery, it is possible that transgene expression might persist for more than a month in this model. Therefore the present HVJ-envelope vector confers an efficient and relatively safe gene transfer technology toward pulmonary vascular disease in rats.

The present study therefore demonstrated that lung cell transduction with ANP by using the present vector ameliorated pulmonary hypertension in rats. These findings are consistent with previous reports showing that respiratory cell transduction with various vasoactive peptides ameliorated pulmonary vascular diseases in rats with pulmonary hypertension but without increased plasma levels of the transduced peptides. ${ }^{24,25}$ Because a previous study showed that cells transfected with the present construct, pUC/rat ANP, release biologically active ANP peptide into the cell culture medium, ${ }^{13}$ it is possible that ANP produced in the transduced epithelial cells exerts paracrine effects on pulmonary vascular vasoreactivity and remodeling and thereby ameliorates pulmonary vascular diseases in rats. ${ }^{24}$ ANP plays a pivotal role in counteracting the mechanisms involved in the development of pulmonary hypertension. The rationale for therapeutic supplementation of ANP against pulmonary hypertension is 2-fold: (1) pulmonary vasodilator responses to ANP are preserved or exaggerated in pulmonary hypertension, ${ }^{9,10}$ and (2) treatments that increase ANP levels, whether by means of ANP infusion or neutral endopeptidase blockade, inhibit the development of pulmonary hypertension and vascular diseases in rats exposed to chronic hypoxia. ${ }^{11,12}$ Therefore consistent with a previous study by Louzier and colleagues, ${ }^{24}$ ANP gene transfer could be an effective treatment strategy against pulmonary hypertension. Clinically, compared with ANP infusion or neutral endopeptidase blockade, ANP lung transfection has less systemic effects and could be clinically modulated by sildenafil administration. ${ }^{26}$ Considering the very short-term effects (10-15 minutes) of ANP inhalation in human subjects, ${ }^{27}$ gene transfer 
approaches with the present vector might be one of the strategies with the longer duration of ANP effects. With respect to the mechanisms, it is interesting to speculate that ANP might inhibit smooth muscle elastase, as well as dilate pulmonary vessels, by increasing cyclic guanosine monophosphate levels because vascular elastase plays a pivotal role in the development of hypoxia-induced pulmonary hypertension and can be inhibited by cyclic guanosine monophosphate increase. $^{20,28}$

Taken together, HVJ-envelope vector could be a novel nonviral vector system that could be applicable to the treatment of pulmonary hypertension. ANP gene transfer to the lungs by using the present vector, which has recently become commercially available, could be a promising therapeutic approach against pulmonary hypertension.

We thank Joji Morita, Department of Pathology II, Mie University School of Medicine, Mie, Japan, for technical assistance with histologic analysis.

\section{References}

1. Newman KD, Dunn PF, Owens JW, Schulick AH, Virmani R, Sukhova G, et al. Adenovirus-mediated gene transfer into normal rabbit arteries results in prolonged vascular cell activation, inflammation, and neointimal hyperplasia. J Clin Invest. 1995;96:2955-65.

2. Gauldie J, Graham F, Xing Z, Braciak T, Foley R, Sime PJ. Adenovirusvector-mediated cytokine gene transfer to lung tissue. Ann N Y Acad Sci. 1996;796:235-44.

3. Crystal RG, Mastrangeli A, Sanders A, Cooke J, King T, Gilbert F, et al. Evaluation of repeat administration of a replication deficient, recombinant adenovirus containing the normal cystic fibrosis transmembrane conductance regulator cDNA to the airways of individuals with cystic fibrosis. Hum Gene Ther. 1995;6:667-703.

4. Piedimonte G, Pickles RJ, Lehmann JR, McCarty D, Costa DL, Boucher RC. Replication-deficient adenoviral vector for gene transfer potentiates airway neurogenic inflammation. Am J Respir Cell Mol Biol. $1997 ; 16: 250-8$.

5. Cornetta K, Morgan RA, Anderson WF. Safety issues related to retroviral-mediated gene transfer in humans. Hum Gene Ther. 1991;2:5-14.

6. Kearns WG, Afione SA, Fulmer SB, Pang MC, Erikson D, Egan M, et al. Recombinant adeno-associated virus (AAV-CFTR) vectors do not integrate in a site-specific fashion in an immortalized epithelial cell line. Gene Ther. 1996;3:748-55.

7. Kaneda $Y$, Nakajima $T$, Nishikawa $T$, Yamamoto S, Ikegami $H$, Suzuki N, et al. Hemagglutinating virus of Japan (HVJ) envelope vector as a versatile gene delivery system. Mol Ther. 2002;6:219-26.

8. Nagaya N, Yokoyama C, Kyotani S, Shimonishi M, Morishita R, Uematsu $\mathrm{M}$, et al. Gene transfer of human prostacyclin synthase ameliorates monocrotaline-induced pulmonary hypertension in rats. Circulation. 2000;102:2005-10.

9. Mitani Y, Maruyama J, Yokochi A, Maruyama K, Yoshimoto T, Naruse M, et al. Modulated vasodilator responses to natriuretic peptides in rats exposed to chronic hypoxia. Eur Respir J. 2000;15:400-6.

10. Jin HK, Yang RH, Thornton RM, Chen YF, Jackson R, Oparil S. Atrial natriuretic peptide lowers pulmonary arterial pressure in hypoxiaadapted rats. J Appl Physiol. 1988;65:1729-35.

11. Jin H, Yang RH, Chen YF, Jackson RM, Oparil S. Atrial natriuretic peptide attenuates the development of pulmonary hypertension in rats adapted to chronic hypoxia. J Clin Invest. 1990;85:115-20.
12. Winter RJ, Zhao L, Krausz T, Hughes JM. Neutral endopeptidase 24.11 inhibition reduces pulmonary vascular remodeling in rats exposed to chronic hypoxia. Am Rev Respir Dis. 1991;144:1342-6.

13. Morishita R, Gibbons GH, Pratt RE, Tomita N, Kaneda Y, Ogihara T, et al. Autocrine and paracrine effects of atrial natriuretic peptide gene transfer on vascular smooth muscle and endothelial cellular growth. J Clin Invest. 1994;94:824-9.

14. Tsujie M, Isaka Y, Ando Y, Akagi Y, Kaneda Y, Ueda N, et al. Gene transfer targeting interstitial fibroblasts by the artificial viral envelopetype hemagglutinating virus of Japan liposome method. Kidney Int. 2000;57:1973-80.

15. Chomczynski P, Sacchi N. Single-step method of RNA isolation by acid guanidinium thiocyanate-phenol-chloroform extraction. Anal Biochem. 1987;162:156-9.

16. Yamanaka M, Greenberg B, Johnson L, Seilhamer J, Brewer M, Friedemann $\mathrm{T}$, et al. Cloning and sequence analysis of the cDNA for the rat atrial natriuretic factor precursor. Nature. 1984;309:719-22.

17. Sawada H, Mitani M, Maruyama J, Jiang BH, Ikeyama Y, Dida FA, et al. A nuclear factor $-\kappa \mathrm{B}$ inhibitor pyrrolidine dithiocarbamate ameliorates pulmonary hypertension in rats. Chest. 2007;132:1265-74.

18. Mitani Y, Maruyama K, Sakurai M. Prolonged administration of L-arginine ameliorates chronic pulmonary hypertension and pulmonary vascular remodeling in rats. Circulation. 1997;96:689-97.

19. Maruyama J, Maruyama K, Mitani Y, Kitabatake M, Yamauchi T, Miyasaka K. Continuous low-dose NO inhalation does not prevent monocrotaline-induced pulmonary hypertension in rats. Am J Physiol Heart Circ Physiol. 1997;272:H517-24.

20. Maruyama K, Ye CL, Woo M, Venkatacharya H, Lines LD, Silver MM, et al. Chronic hypoxic pulmonary hypertension in rats and increased elastolytic activity. Am J Physiol Heart Circ Physiol. 1991;261: H1716-26.

21. Shimamura M, Morishita R, Endoh M, Oshima K, Aoki M, Waguri S, et al. HVJ-envelope vector for gene transfer into central nervous system. Biochem Biophys Res Commun. 2003;300:464-71.

22. Nakamura H, Kimura T, Ikegami H, Ogita K, Koyama S, Shimoya K, et al. Highly efficient and minimally invasive in-vivo gene transfer to the mouse uterus using haemagglutinating virus of Japan envelope vector. Mol Hum Reprod. 2003;9:603-9.

23. Saeki Y, Wataya-Kaneda M, Tanaka K, Kaneda Y. Sustained transgene expression in vitro and in vivo using an Epstein-Barr virus replicon vector system combined with HVJ liposomes. Gene Ther. 1998;5: 1031-7.

24. Louzier V, Eddahibi S, Raffestin B, Deprez I, Adam M, Levame M, et al. Adenovirus-mediated atrial natriuretic protein expression in the lung protects rats from hypoxia-induced pulmonary hypertension. Hum Gene Ther. 2001;12:503-13.

25. Champion HC, Bivalacqua TJ, Toyoda K, Heistad DD, Hyman AL, Kadowitz PJ. In vivo gene transfer of prepro-calcitonin gene-related peptide to the lung attenuates chronic hypoxia-induced pulmonary hypertension in the mouse. Circulation. 2000;101:923-30.

26. Michelakis ED, Tymchak W, Noga M, Webster L, Wu XC, Lien D, et al. Long-term treatment with oral sildenafil is safe and improves functional capacity and hemodynamics in patients with pulmonary arterial hypertension. Circulation. 2003;108:2066-9.

27. Angus RM, Millar EA, Chalmers GW, Thomson NC. Effect of inhaled thiorphan, a neutral endopeptidase inhibitor, on the bronchodilator response to inhaled atrial natriuretic peptide (ANP). Thorax. 1996;51: 71-4.

28. Mitani Y, Zaidi SH, Dufourcq P, Thompson K, Rabinovitch M. Nitric oxide reduces vascular smooth muscle cell elastase activity through cGMP-mediated suppression of ERK phosphorylation and AML1B nuclear partitioning. FASEB J. 2000;14:805-14. 